\title{
Carnets
}

Revue électronique d'études françaises de l'APEF

Deuxième série - 17 | 2019

Lîlle : prisme de la connaissance ou reconnaissance du monde

\section{La dualité de l'étendue : la représentation des Kerguelen de Jean-Paul Kauffmann}

\section{Silvia Baage}

\section{(2) OpenEdition}

\section{Journals}

Édition électronique

URL : https://journals.openedition.org/carnets/10243

DOI : $10.4000 /$ carnets. 10243

ISSN : 1646-7698

Éditeur

APEF

Référence électronique

Silvia Baage, "La dualité de l'étendue : la représentation des Kerguelen de Jean-Paul Kauffmann », Carnets [En ligne], Deuxième série - 17 | 2019, mis en ligne le 30 novembre 2019, consulté le 28 juin 2022. URL : http://journals.openedition.org/carnets/10243 ; DOI : https://doi.org/10.4000/carnets. 10243

Ce document a été généré automatiquement le 29 septembre 2020.

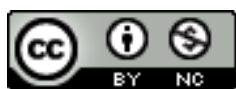

Carnets est mis à disposition selon les termes de la licence Creative Commons - Atribution - Pas d'utilisation commerciale 4.0 International. 


\title{
La dualité de l'étendue : la représentation des Kerguelen de Jean-Paul Kauffmann
}

\author{
Silvia Baage
}

1 Jean-Paul Kauffmann, accorde une place importante à l'expérience de la claustrophobie et de l'enfermement. Ces thèmes figurent également dans les textes consacrés aux voyages aux îles proches et lointaines, notamment dans La Chambre noire de Longwood : le voyage à Sainte-Hélène (Kauffmann, 1997), ouvrage qui relate les derniers jours de Napoléon Bonaparte en exil, ou encore, L'Arche des Kerguelen : voyage aux îles de la Désolation $^{1}$ (Kauffmann, 2002), le récit d'un voyage aux TAAF, terres australes et antarctiques françaises, qui explore les traces des grands explorateurs français dans une région sans population permanente. La figure de l'île-prison est présente dans les deux textes mais dans le cas du voyage à l'archipel des Kerguelen, la volonté de documentation et d'exotisme mérite une analyse en profondeur.

De fait, les contrées insulaires fortement isolées, soumises à l'hostilité des climats rudes, font souvent état d'une grande disparité dans les études concernant les récits de voyage. Or, ce qui est fascinant, c'est la situation de cette figure de l'île-prison dans un réseau d'archipels dispersés entre les $40^{\circ}$ rugissants et les $50^{\circ}$ hurlants ainsi que le fait qu'elle inspire des rêves et des cauchemars depuis plusieurs siècles. Dotées d'une flore et d'une faune tout à fait insolites qui affirment une irréductible singularité, les TAAF constituent un ensemble archipélique exceptionnel de l'espace outre-mer français, " habitées seulement par quelques dizaines de scientifiques ou une poignée de militaires s'y relayant célibataires » (Gay, $2008: 162)$. L'archipel des Kerguelen fait partie des cinq districts taafiens, et représente, chez Kauffmann, un cas tout à fait particulier de volonté de documentation et d'exotisme dont on ne peut pas ignorer la dimension intertextuelle. Pour Jacqueline Dutton, la métaphore d'« imperial ice $»^{2}$ explique le croisement entre les fragments textuels d'anciens récits de voyage imbriqués dans les passages visant la description du terrain. Mais il convient de regarder de plus près le rapprochement entre les bribes intertextuelles et la description de la nature qui, en 
effet, prennent un double sens dans la mesure où cette dualité inquiétante dépasse le cadre de la réflexion sur l'histoire de l'empire.

3 Pour ce faire, nous nous proposerons de compléter l'approche diachronique de Dutton avec une approche synchronique des motifs d'exotisme de Kauffmann à l'ère de la mondialisation où la terre est prétendument déterritorialisée. Notre approche synchronique insistera davantage sur la volonté de documentation et d'exotisme dans son rapport à une forme narrative particulière de l'aventure exotique que Jean-Marc Moura a conçue comme voyage rétrospectif : « ces romans bien connus qui sont à la fois fortement réflexifs et qui pourtant paradoxalement se réfèrent à des personnages et à des événements historiques" (Moura, 2003 : 38). Analyser L'Arche des Kerguelen de Kauffmann à travers les caractéristiques du voyage rétrospectif identifiées par Moura nous permettra de nous interroger sur la dualité de la lecture et de l'espace kerguelénien, que cela soit de la glace ou des paysages sauvages. Il s'agira donc de relire L'Arche des Kerguelen sous l'angle de ces dualités.

\section{Entre gloire et malheur : la dualité du projet de voyage dans les terres lointaines}

Dans un premier temps, L'Arche des Kerguelen: Voyage aux îles de la Désolation raconte le périple de Kauffmann qui se propose de suivre les traces des explorateurs pour découvrir les Kerguelen. C'est ainsi que dans l'ensemble, les quatre chapitres présentent la dimension géographique du voyage, à partir de l'Ile de la Réunion, point de départ du navire ravitailleur, le Marion Dufresne. Chacune des quatre parties met en valeur un éloignement de plus en plus important dont nous retenons particulièrement le passage de la convergence subantarctique ainsi que l'arrivée à Port-aux-Français de la première partie, les déplacements et raids de la deuxième partie, le séjour à Port Jeanne d'Arc de la troisième partie et dans la dernière partie, l'exploration des alentours de Port-Christmas qui s'achève sur l'annulation de la manip, parler taafien pour désigner une excursion hors de la base $\mathrm{b}^{3}$. Si la dimension géographique des descriptions traduit la progression de l'auteur dans l'espace, il convient de s'interroger sur la place que prennent les rêveries et l'anticipation du voyageur qui ne pense qu'à voir l'arche des Kerguelen, le point d'accès des Kerguelen le plus difficile à atteindre. Édouard Glissant a bien résumé le défi du voyageur face à l'isolement extrême, en l'occurrence, l'île de Pâques : « Il y avait donc dans le monde des lieux qui se trouvaient tellement isolés, (...) qu'il fallait pour y atteindre disposer de toutes ses facultés physiques et espérer, pour pouvoir y rester quelque peu, ne pas succomber à des faiblesses d'esprit » (Glissant, $2007: 10$ ), propos sur lesquels nous reviendrons plus tard. Nous constatons qu'en effet le récit de Kauffmann est rythmé par son inquiétude face aux conditions climatiques qui feront réussir ou échouer son projet de voir l'arche des Kerguelen, au large de Port-Christmas.

Le voyageur a beau insister sur la réhabilitation des noms oubliés, objectif qu'il atteint d'ailleurs avec l'installation de la plaque en l'honneur des frères Bossière. Or, la dualité du projet de ce voyage s'annonce dès le début. Pour commencer, nous retrouvons dans le titre la double appellation de l'archipel, au carrefour de la gloire et du déshonneur : d'une part, l'archipel des Kerguelen désigne un espace ayant trouvé ses origines plus ou moins glorieuses dans la lignée des grands explorateurs français, de Kerguelen aux frères Bossière, d'autre part, cette double appellation nous rappelle les conséquences 
fatales de cette découverte problématique pour les aventuriers du bout du monde qui s'y perdent aussi bien physiquement que mentalement. S'ajoute également l'Arche comme image emblématique de cet espace lointain et isolé : en tant que projet architectural façonné par la Nature, l'arche ressemble à une entrée courbée, voire une voûte, si bien que cette porte mythique franchit un espace qui trouve tout son sens dans sa dimension symbolique. L'arche représente, entre autres, « la demeure protégée par Dieu (Noé) et sauvegardant les espèces (...) Elle revêt le triple sens symbolique de nouvelle alliance, qui est universelle et éternelle ; de nouvelle présence, qui est réelle ; de nouvelle arche de salut, non plus contre le déluge, mais contre le péché » (Chevalier et Cheerbrant, 1982 : 73), symbolisme pourtant inquiétant si on considère les drames oubliés qui se sont déroulés aux Kerguelen; de plus, la partie supérieure de cette arche, la voûte, couvre un espace céleste, celui de «l'union du ciel et de la terre » (Chevalier et Cheerbrant, 1982 : 1027). Quels rôles prennent ces dénotations et connotations dans l'ensemble du texte pour décrire le sens de cette aventure exotique pour Kauffmann?

Dutton a insisté sur le lien entre la littérature et l'empire à tel point qu'elle s'attache à la construction antithétique du récit, autrement dit, elle se focalise sur la juxtaposition des moments passés de gloire et de souffrance des grands explorateurs comme intertexte afin de les comparer aux expériences du voyageur à l'époque moderne. Certes, ce voyageur masculin fait preuve d'une grande volonté de posséder, de nommer et de classifier le terrain inhabité et sauvage, à l'instar des explorateurs de l'époque coloniale, comme le montrent de nombreux fragments intertextuels tout au long du texte. Dans ce sens-là, la lecture de ces fragments intertextuels signifie, comme l'explique Dutton, un hiatus dans la narration du périple qui permettrait de cacher, en quelque sorte, la déception et l'échec de Kauffmann adulte face à l'objet de son désir, comme le suggère d'ailleurs l'épigraphe également citée par Dutton : " N'as-tu pas comme moi rêvé, enfant, devant ton atlas grand ouvert à une de ses pages les plus remplies d'azur, de cette lointaine et pourtant nôtre, antarctique et française, Kerguelen » (Dutton, 2009 : 374).

7 Cependant, le rapport entre le passé colonial et le présent de la narration prend un autre sens si on considère la lecture non pas comme une volonté de documentation et de rappel au passé glorieux ${ }^{4}$ mais comme une forme contemporaine d'exotisme qui relève de la métafiction du voyage ${ }^{5}$ dans le sens où Moura entend ce terme. Ainsi, pour Moura, il s'agit d'insister sur d'autres aspects historiques qui dépassent la réflexion sur les valeurs de l'empire, à savoir, les possibilités du voyage à l'ère moderne :

Les récits (...) confrontent en réalité différentes formes du voyage pour engager une méditation sur le sens du périple et de l'exotisme en un temps où la connaissance historique (...) [s'est] extra-ordinairement développée (...), en un moment où nous savons mieux que jamais ce qui nous est accordé et ce qui nous est désormais interdit par le voyage (Moura, $2003: 39$ ).

Il s'agit donc de comprendre comment Kauffmann se situe par rapport au privilège de découvrir cet espace ultrapériphérique dans le sens où Gay entend ce terme, à l'encontre de Glissant qui se résigne à fréquenter l'île de Pâques comme rêveur lointain, " par les commentaires que je ferais de ce qu'elle [Sylvie] enverrait et de ce qu'elle rapporterait »(Glissant, 2007 : 9). Kauffmann, par contre, a la chance de fréquenter le terrain mais sa volonté d'exotisme reste ambiguë. Il insiste à plusieurs reprises sur une sorte de continuité territoriale de l'archipel « aussi verdoyant que les prairies de [la] Bretagne » $(A F, 15-16)$, avec un ensoleillement « identique (...) à celui de Biarritz » $(A K, 102)$ et ses baraquements qui forment un « fragile village de banlieue 
ouvert à tout vent (...). Si loin de tout, si chétif au milieu de cette nature bizarre. Celleci a l'air 'retardée' » $(A K, 33)$. Cette remarque sur le retard de la nature archipélagique prend un sens mythique qui renvoie au symbolisme de l'unité perdue de l'arche : « Le paysage semble porter la trace de l'imperfection originelle. La création s'est arrêtée ici au cinquième jour avec les poissons et les oiseaux " (AK, 164). Le recours au provincialisme de la France profonde ${ }^{6}$ est ainsi embelli du charme désuet de la Genèse pour traduire le dépaysement du voyageur moderne face aux Kerguelen où le sens du péché originel et du paradis mythique se confondent. C'est ainsi que les bribes intertextuelles prennent tout leur sens, vu que Kauffmann se sert de l'intertexte afin d'expliquer le caractère insolite de son expérience : «Dans quelle catégorie doit-on les ranger ? Depuis mon arrivée, je m'aperçois qu'il est difficile de décrire et même de concevoir les Kerguelen. Tous les voyageurs ont éprouvé ce sentiment d'impuissance » $(A K, 43)$. Force est de constater que se met en place une tension entre le but recherché de l'aventure extrême au bout du monde et sa reconstitution romanesque qui oblige l'auteur à avouer que l'« on patauge dans la souille des Kerguelen, on est dans le concret. Pourtant, les Kerguelen ne sont pas concrètes'. Elles échappent au réel, elles ne sont assujetties qu'à l'imagination " $(A K, 151)$. La réflexion sur les motifs du voyage rétrospectif nous permet donc de passer du lien entre la littérature et l'empire à une analyse de la dualité du sens de l'aventure exotique que l'on attribue au roman postmoderne. Il s'agira donc d'aborder la lecture dans une dimension synchronique.

Les Kerguelen, une tour de Babel?

Nous confirmons avec Dutton que Kauffmann accorde une importance particulière à l'acte de lire mais il convient d'insister avec Moura " qu'il ne faut pas confondre, comme remarquait André Breton, les livres qu'on lit en voyage et les livres qui font voyager " (Moura, $2003:$ 32). En effet, chez Kauffmann, les Kerguelen sont l'endroit privilégié d'une lecture en voyage ainsi que l'objet d'une lecture avide de livres qui font voyager car, après tout, l'isolement extrême permet au voyageur de s'adonner aux lectures. Mais avant de partir, Kauffmann avoue que malgré toutes ses lectures qui le faisaient voyager aux Kerguelen, il n'est pas parvenu à mieux comprendre cet espace :

Dans ma recherche, je me suis aperçu que les faits entourant la découverte des Kerguelen, puis son exploration entre 1790 et 1914, n'expliquent absolument rien. Aucune continuité. Baleiniers et missions scientifiques se sont presque toujours succédé en s'ignorant. Je me suis demandé parfois s'il s'agissait du même pays ( $A K$, 19).

Effectivement, l'exégèse extra-diégétique de textes en relation avec l'archipel des Kerguelen révèle une incohérence à laquelle le voyageur n'est pas réfractaire :

La Désolation a fait l'objet d'études savantes sur le magnétisme, la sismologie, la biologie animale, etc. Mais c'est la vue d'ensemble qui fait défaut. Tout a été recensé et cependant les Kerguelen gardent leur mystère. (...) Rigoureux mais toujours incomplets, ils rappellent la tour de Babel. Chacun parle la langue de sa discipline et ignore celle du voisin $(A K, 53)$.

11 Il n'est donc pas anodin de remarquer que malgré toute volonté de documentation scientifique, il est de nombreux récits fragmentés voire fragmentaires qui font écho à la condition postmoderne au sens où Jean-François Lyotard entend ce terme. C'est précisément le rapprochement entre le fragmenté et le fragmentaire qui nous 
interpelle ici dans son rapport au sens de l'aventure exotique aux Kerguelen : dans cet espace réservé à la science, serait-il possible de résoudre le mystère des Kerguelen si toutes les disciplines, en passant des sciences humaines aux belles lettres, s'associaient ? L'approche littéraire saurait-elle créer le pont pour envisager un tel projet ? Ou bien, avec son attachement au mythe, à l'instar de Babel, Kauffmann n'évoque-t-il pas aussi le risque du désir de toute connaissance, en l'occurrence, celle du mystère des Kerguelen?

Quant aux livres lus en voyage, il est inutile de repasser sur le corpus du tissu intertextuel déjà développé par Dutton mais notons toutefois l'attachement de l'auteur aux convergences et aux divergences entre les textes qu'il cite, notamment, les liens tissés dans les témoignages des explorateurs et les robinsonnades ainsi que le guide de Gracie Delépine qui, pour sa part, ne manque pas de se référer aux explorateurs comme Kerguelen, Edgar Aubert de la Ruë et Raymond Rallier du Baty. Une telle accumulation de bribes intertextuelles représente pour Moura la première caractéristique du voyage rétrospectif qu'il décrit comme " fragmentation saisie du passé par fragments à partir desquels s'organise la rêverie » (Moura, 2003 : 38). Mais il ne faut pas oublier de situer cette rêverie, inspirée par les fragments textuels, sur le plan synchronique pour montrer l'ambiguïté du sens de la lecture. En fait, Kauffmann commente les titres et passages qu'il cite. La conclusion du texte est révélatrice à cet égard. Aveuglé par ses lectures, l'auteur ne pense qu'à atteindre l'arche et le voyage se termine sur un échec personnel : « Retour en France. Je découvre que l'arche n'existe plus. Elle a été détruite entre 1909 et 1914. Si j'avais lu plus attentivement le récit du second voyage de Rallier du Baty, j'aurais appris que de l'arche ne subsistent aujourd'hui que 'deux colonnes semblables aux tours de Notre-Dame' " $(A K, 254)$. Cette révélation douloureuse est complétée, entre autres, par l'aveu de l'auteur d'avoir commis les mêmes erreurs lorsqu'il lisait, plus jeune, si bien que la chute du redoutable héros du roman L'Ancre de miséricorde le frappait. Cette frustration illustre les périls d'une lecture distraite voire l'ignorance du lecteur : « je n'ai pas souvenir d'avoir goûté alors les séductions de l'ambiguïté, ni même d'avoir compris l'inquiétant jeu de cache-cache qui se poursuit jusqu'à la révélation finale. J'ignorais que ce divertissement douloureux et inexplicable se nommât la littérature " $(A K, 93)$. Quel rôle les textes littéraires jouent-t-ils dans l'ensemble du voyage que propose Kauffmann?

\section{米}

13 Le rapprochement d'un lieu de passage hors du commun : la dualité de l'étendue

Il n'est pas difficile de relever les similarités entre le caractère douloureux et inexplicable de la lecture et celui du voyage qu'entreprend Kauffmann :

Je sais bien qu'au bout de ma vie, seule une dizaine de livres auront compté. Les autres ? Paysages aperçus, vite oubliés, plaisirs fugitifs. Le fragment, la ferveur, l'étourdissement du moment, le droit de se contredire, le nomadisme : illusion des années 70. Je comprends mieux pourquoi les Kerguelen me plaisent. Elles contredisent cet éparpillement (AK, 64-65).

Le constat de l'auteur révèle le sens du voyage à l'ère moderne : le tissu intertextuel est moins utile dans son rapport à la réflexion sur la rêverie du voyageur au sujet de l'empire mais plutôt, dans son rapport au fragment et au fragmentaire. En effet, il convient de comparer cette révélation à l'avènement d'une littérature voyageuse placée sous l'égide de Michel Le Bris et de Kenneth White qui prônent l'immensité et l'étendue 
pour baliser le chemin, au xxI ${ }^{e}$ siècle, pour une forme de récit de voyage que Graham Huggan décrit comme Extreme Pursuits (Huggan, 2009). Pourtant, le récit de Kauffmann insiste sur l'impossibilité du voyage, comme nous venons de le voir, pour faire preuve de ce que Charles Forsdick appelle la "tyrannie de l'étendue et de l'immensité " (Forsdick, $2005:$ 184) des années 90. Cet isolement extrême des TAAF est sans aucun doute fortement agrémenté par la fragmentation de l'archipel kerguelénien dont l'île principale est « entourée de plus de 300 îles et îlots satellites, pour la plupart très proches de l'île principale. Les côtes sont dans leur ensemble extrêmement découpées avec quelques grandes golfes et de nombreuses baies secondaires $»^{7}$. Le voyageur de l'espace kerguelénien risque ainsi d'être dérouté par l'étendue de l'espace et ce n'est pas la lecture des livres qui le sauve : "Quand (...) on ne peut plus s'appuyer que sur ce qui existe à l'intérieur de soi-même, serait-ce le livre qu'on n'a pas choisi qui sauve ? Robinson Crusoé disposait d'une Bible. (...) Il se plaisait à l'ouvrir au hasard et interprétait lumineusement le moindre verset » $(A K, 114)$. Il convient de préciser que le sens même du voyage dans l'espace kerguelénien reste ambivalent, comme l'a montré en 2015 l'ancien administrateur supérieur des TAAF, François Garde, lorsqu'il a traversé l'archipel, de l'extrême nord à l'extrême sud, afin de vaincre l'étendue et l'immensité des Kerguelen en liant les "traits rageurs sur la carte, qui ne se rejoignaient pas, comme des ratures » (Garde, $2018: 17$ ), exploit physique et mental plutôt qu'effort intellectuel qu'il relate dans Marcher à Kerguelen. Ce genre de voyage " en terrain hostile, sans chemin ni répit ni confort » (Garde, $2018: 16)$ où l'étendue oblige car « il n'y a pas d'autre issue que d'aller jusqu'au bout » (Garde, $2018: 16$ ) s'oppose à un autre type de voyage qu'évoque Charles Forsdick pour montrer l'avènement de nouvelles formes du récit de voyage après les années 90 qui s'orientent vers les déplacements du voyageur de l'immédiat non pas dans les franges de l'empire, mais dans une France profonde, repliée sur elle-même. C'est dans ce sens-là qu'il faut comprendre la dualité de l'espace kerguelénien chez Kauffmann car en dépit de la frustration du rendez-vous manqué avec l'arche, l'auteur réussit à dénicher d'autres trésors propres à l'espace taafien tels que les archives des rapports que personne ne lit et où il trouve cette observation: "J'en ai connu le meilleur et le pire. Le meilleur, la beauté sauvage et grandiose de ces îles. Le pire, les hommes avec leurs mesquineries déplacées dans ce cadre » (AK, 232).

15 Or, ce régionalisme ainsi que les rites initiatiques des hivernants indiquent qu'en effet, l'espace kerguelénien se profile comme un lieu de passage hors du commun dans le sens où Alexandra Marois l'a décrit : "Séjourner aux Kerguelen représente donc un moment de reconstruction d'un espace-temps différent, en rupture avec le quotidien » (Marois, $2003:$ : 81). À l'instar du voyageur moderne qui se déplace grâce aux guides de voyage, le narrateur explique son dépaysement dans cette immensité de l'espace taafien ainsi :

je ne suis qu'un touriste. Mais je ne veux rien manquer : je 'fais' les Kerguelen comme d'autres 'font' les Cyclades ou les églises (...). L'inélégance du mot me convient. On 'fait' un pays comme on exécute un ouvrage d'après un plan ou un calendrier (...). En racontant ce pays, je sais bien que je lui ôte un peu de son intégrité (AK, 94-95).

16 Cette remarque n'est pas sans évoquer le commentaire de François Graveline qui souligne fort bien dans sa présentation des Naufragés de l'Aventure de Guillaume Lesquin que les TAAF s'avèrent les "îles où l'homme n'a tout simplement pas sa place " (Graveline, $2010: 7$ ). 
17 C'est ainsi que le récit de Kauffmann prend une position ambivalente par rapport au propos de Moura qui prône le voyage rétrospectif comme « une méditation littéraire sur ce qui peut désormais échapper au périple de masse. Elle est ainsi réflexion critique sur la force d'exotisme du voyage, mouvement d'exploration du rapport de l'homme à la terre et affirmation de la puissance de la littérature » (Moura, $2003: 40$ ). Il semblerait que Kauffmann conjugue cette "force d'exotisme du voyage " aussi bien que « la puissance de la littérature " à sa façon. Certes, la question de l'exotisme est présente car l'auteur avoue que "Partir aux Kerguelen n'est pas chose aisée. Les îles de la Désolation passent pour être le point le plus isolé du globe. (...) L'éloignement des Kerguelen est l'une des dernières curiosités d'une époque qui se flatte d'abolir l'espace et le temps » $(A K, 17)$. Mais la dualité de l'exotisme et du voyage se manifeste dans la présence humaine dans un espace à la fois spirituel et fragile. Cette dualité renvoie au concept de l'exotisme dans le sens où Moura entend ce terme : « Au fond, 'exotisme' est l'un des noms modernes de la nostalgie. Lié à l'inachèvement de la condition humaine, il réside dans l'aspiration inguérissable à trouver le salut dans une autre région du réel "(Moura, $2003:$ 18). Il en va de même pour l'acte de lire, surtout la lecture en voyage, qui risque de dérouter l'expérience du voyageur à cause d'une accumulation de sources que Charles Forsdick décrit comme une mosaïque d'étoffes (Forsdick, 2005 : 141). La composition du texte en bribes fragmentées traduit en effet le progrès difficile du voyageur et l'impossibilité du voyage qui s'explique non pas à travers le problème de l'isolement extrême mais plutôt à travers le défi de la description du caractère insolite de l'aventure extrême : "Intermédiaire entre l'ailleurs et le partout, la Désolation se situe dans un troisième lieu. Kerguelen ne croyait pas si bien dire quand il l'appelait 'troisième monde'. [... Les Kerguelen] sont un défi au pittoresque, à l'exotisme, au voyage " $(A K, 68)$. Les Kerguelen tendent donc vers l'irreprésentable, autre caractéristique du voyage rétrospectif selon Moura, pour faire écho, en quelque sorte, au droit à l'opacité, dans le sens où Glissant entend ce terme. Si la mosaïque d'étoffes reste foncièrement littéraire chez Kauffmann en 1993, elle prendra d'autres formes pour les voyageurs de l'immédiat du $\mathrm{XxI}^{\mathrm{e}}$ siècle, dont Glissant, qui, dans son récit de voyage à l'île de Pâques, mélange de façon stratégique de nombreux supports visuels et médiatiques pour créer une mosaïque d'étoffes qui sort du domaine strictement littéraire afin de faire connaître une autre île mystérieuse de l'autre bout du monde.

\section{BIBLIOGRAPHIE}

CHEVALIER, Jean, CHEERBRANT, Alain (1982 [1969]). Dictionnaire des symboles. Paris : Éditions Laffont et Éditions Jupiter.

DUTTON, Jacqueline (2009). « Imperial Ice? The influence of Empire on contemporary French and British Antarctic travel writing ", Studies in Travel Writing. Papers from the Essex Symposium on 'writing travels', $\mathrm{n}^{\circ} 13.4, \mathrm{pp} .369-380$.

FORSDICK, Charles (2005). Travel in Twentieth Century French and Francophone Cultures: The Persistence of Diversity. Oxford : Presses universitaires d'Oxford. 
FULIGNI, Bruno (2014). Le Tour du monde des terres françaises oubliées. Paris : Éditions du trésor.

GARDE, François (2018). Marcher à Kerguelen. Paris : Gallimard.

GAY, Jean-Christophe (2008). L'Outre-mer français : Un espace singulier. Paris : Belin.

GLISSANT, Édouard, SÉMA, Sylvie (2007). La Terre magnétique : Les errances de Rapa Nui, l'île de Pâques.

Paris : Seuil.

GRAVELINE, François (2010). « Une image de la destinée humaine », in Les Naufragés de l'Aventure.

Lassay-les-Châteaux : Éditions Nicolas Chaudun, pp. 5-15.

HUGGAN, Graham (2009). Extreme Pursuits : Travel/writing in an Age of Globalization. Ann Arbor :

Presses universitaires du Michigan.

KAUFFMANN, Jean-Paul (2002 [Flammarion, 1993]). L'Arche aux Kerguelen : Voyage aux îles de la

Désolation. Paris : Éditions de la Table Ronde.

KAUfFMANN, Jean-Paul (1997). La Chambre noire de Longwood: Le Voyage à Sainte-Hélène. Paris : La

Table Ronde.

MAROIS, Alexandra (2003). Les Iles Kerguelen : Un monde exotique sans indigène, Étude ethnologique

d'une communauté transitoire dans un espace clos. Paris : L'Harmattan.

MOURA, Jean-Marc (2003). L'Exotisme et lettres francophones. Paris : Presses Universitaires de France.

\section{NOTES}

1. Toutes les citations de ce texte seront suivies dans l'article par l'abréviation AK suivie de la pagination.

2. « (...) contemporary travel narratives (...) may on the one hand integrate classic examples of the imperial gaze, and on the other hand, reinvent the contact zone. In the absence of opposing cultural influences, the ice itself takes on an inherently anachronistic role, freezing in time and nature the characteristics and values of the French (...) Empire (...) like an environmental monument to imperialism. " [ "Il se peut que les récits de voyage contemporains incorporent d'une part des exemples classiques du regard impérial et d'autre part, ils réinventent la zone de contact. En l'absence de forces culturelles opposées, c'est la glace qui prend un rôle fondamentalement anachronique, gardant une forme figée des caractéristiques et valeurs de l'Empire français comme un monument écologique de l'impérialisme. »] (Dutton, 2009 : 379).

3. Voir à ce sujet l'étude d'Alexandra Marois, Les Iles Kerguelen : Un monde exotique sans indigène, Étude ethnologique d'une communauté transitoire dans un espace clos.

4. Voir à ce propos Tour du monde des terres françaises oubliées de Bruno Fuligni qui cherche à faire sortir de l'oubli les taches inconnues des cartes, comme le souligne la quatrième de couverture : « L'empire colonial français est mort, mais le drapeau tricolore flotte encore sur nombre de terres exotiques et bizarres ». (Fuligni, $2014: 11-12$ )

5. « Une forme romanesque singulière pour laquelle la critique française n'a pas encore de nom (...) évoqu[e] des aventures exotiques passées (...). L'exploration critique du voyage passé correspond à un courant attesté un peu partout en Europe. On peut parler à son égard de métafiction du voyage, en référence à une forme centrale du roman postmoderne, la 'historiographic metafiction' mise en évidence par Linda Hutcheon » (Moura, 2003 : 20-21).

6. Alexandra Marois nous rappelle à ce propos la comparaison de Françoise Sylvestre qui décrit l'archipel des Kerguelen comme étant « à peine plus grand comme [que] la Corse » (Marois, 2003 :16). 


\section{RÉSUMÉS}

La critique littéraire s'intéresse rarement aux récits de voyage dans les contrées insulaires ultrapériphériques telles que les TAAF, terres australes et antarctiques françaises. Ce réseau d'archipels dispersés inspire des rêves et des cauchemars depuis des siècles. Dans cette étude, nous nous focaliserons sur L'Arche des Kerguelen : voyage aux îles de la Désolation de Jean-Paul Kauffmann afin d'analyser la représentation des TAAF comme île-prison irreprésentable qui échappe au voyageur moderne, en dépit des textes littéraires qui l'accompagnent.

Literary criticism rarely focuses on travel literature about ultra-peripheral island regions such as the French Southern and Antarctic Lands. This network of scattered archipelagos has triggered dreams and nightmares for centuries. In this study, we will focus on Jean-Paul Kauffmann's Arch of Kerguelen: Voyage to the Islands of Desolation to analyze the representation of the French Southern and Antarctic Lands as an irrepresentable island-prison that escapes the modern traveler, despite the literary texts that accompany him.

\section{INDEX}

Keywords : travel, island-prison, exoticism, intertextuality, fragment

Mots-clés : voyage, île-prison, exotisme, intertextualité, fragment

\section{AUTEUR}

\section{SILVIA BAAGE}

McDaniel College

sbaage[at]gmail.com 